\title{
Supernovae, CMB, and gravitational leakage into extra dimensions
}

\author{
Cédric Deffayet, ${ }^{1, *}$ Susana J. Landau, ${ }^{2, \dagger}$ Julien Raux, ${ }^{3, \uparrow}$ Matias Zaldarriaga, ${ }^{1, \S}$ and Pierre Astier ${ }^{3, \|}$ \\ ${ }^{1}$ Department of Physics, New York University, 4 Washington Place, New York, New York 10003 \\ ${ }^{2}$ Observatorio Astronómico, Universidad Nacional de La Plata, Paseo del Bosque S/N, CP 1900 La Plata, Argentina \\ ${ }^{3}$ LPNHE, CNRS-IN2P3 and Universités Paris VI \& VII, Paris, France
}

(Received 26 January 2002; published 12 July 2002)

\begin{abstract}
We discuss observational constraints coming from CMB and type Ia supernovae for the model of an accelerated universe produced by gravitational leakage into extra dimensions. Our fits indicate that the model is currently in agreement with the data. We also give the equations governing the evolution of cosmological perturbations. Future observations will be able to severely constrain the model.
\end{abstract}

DOI: 10.1103/PhysRevD.66.024019

PACS number(s): 04.50.+h, 98.70.Vc

\section{INTRODUCTION}

Supernovae observations have recently provided evidence that the expansion of the Universe is undergoing a late time acceleration [1-3]. This acceleration can be explained in the framework of standard cosmology by a nonvanishing cosmological constant. Although in agreement with current observations, such an explanation exacerbates the usual cosmological constant problem because it requires an explanation for its very small, but nonzero, value.

One may wish to find alternative explanations for the acceleration, and there are several proposals in the literature. Here we explore a scenario proposed in $[4,5]$, based on the model of Dvali-Gabadadze-Porrati of brane-induced gravity [6]. This proposal explains the observed late time acceleration of the expansion of the Universe through a large scale modification of gravity coming from "leakage" of gravity at large scale into an extra dimension, and without requiring a nonvanishing cosmological constant. The interesting point about this model from a phenomenological perspective is that it is a testable alternative to a cosmological constant model with the same number of parameters. This is in contrast with models of "quintessence" where the equation of state of the new component becomes a free function that needs to be constrained.

In [5] it has been shown that the model was in qualitative agreement with all known cosmological observations. The purpose of this work is to go one step further and quantitatively confront the model with observations of supernovae and the cosmic microwave background (CMB). the dynamics of the background metric of the universe in the model. We first introduce in a few words the brane-induced gravity model of Dvali-Gabadadze-Porrati [6] (see also [7-9]) which provides the framework (Sec. II A). We then discuss the cosmological dynamics for the accelerated solution considered in this paper (Sec. II B). In the following, we
The paper is organized as follows. In Sec. II we discuss

confront the model with the supernovae observations of the Supernova Cosmology Project (SCP) [2] (Sec. III A) and CMB data (Sec. III B).

Our fits indicate that the model is currently in agreement with supernova type Ia (SNIa) and small scale CMB data. One can hope to discriminate the model from standard cosmology using future precision cosmological parameters measurements, but also maybe modifications in the growth of large scale structures.

\section{MODEL DEFINITION AND BACKGROUND DYNAMICS}

In the following subsections we summarize the main features of the model under consideration and study the dynamics of the background metric.

\section{A. Brane-induced gravity models in a few words}

The brane-induced gravity models are a particular class of brane-world models, which can be defined as models where our four dimensional (4D) universe is considered to be a surface (called brane) embedded into a higher dimensional bulk space-time.

Brane world models are inspired by superstring-M theory, and can be regarded as some low energy effective models of more fundamental underlying theories, but have also interest on their own in providing new phenomenological ideas. We will only consider here the case where the bulk is five dimensional (5D). The brane embedding into the bulk is defined by the coordinates $X^{A}\left(x^{\mu}\right)$ of the brane world volume (parametrized by coordinates $x^{\mu}$ ) into the 5D space-time. The dynamics of gravity is governed by the usual 5D Einstein-Hilbert action

$$
S_{E H}=\left.\frac{M_{(5)}^{3}}{2} \int d^{5} X \sqrt{\mid(5)} g\right|^{(5)} R,
$$

†Email address: slandau@fcaglp.fcaglp.unlp.edu.ar

*Email address: raux@in2p3.fr

$\S$ Email address: mz31@nyu.edu

"Email address: pierre.astier@in2p3.fr where $M_{(5)}$ denotes the 5D reduced Planck mass. The bulk 
metric ${ }^{(5)} g_{A B}$ induces through the embedding $X^{A}\left(x^{\mu}\right)$ a metric $g_{\mu \nu}$ on the brane (called induced metric) defined by ${ }^{1}$

$$
g_{\mu \nu}={ }^{(5)} g_{A B} \partial_{\mu} X^{A} \partial_{\nu} X^{B} .
$$

In the above equation, we have put an upper index, ${ }^{(5)}$, on quantities (e.g., the 5D Ricci scalar ${ }^{(5)} R$ or the $5 \mathrm{D}$ metric ${ }^{(5)} g_{A B}$ ) to distinguish them from their $4 \mathrm{D}$ counterparts, depending only on the induced metric (e.g., $R$ or $g_{\mu \nu}$ ).

In the brane-induced gravity models [6-10], the gravitational action contains another term $S_{e h}$, in addition to the 5D Einstein-Hilbert term (1), given by

$$
S_{\text {eh }}=\frac{M_{\mathrm{Pl}}^{2}}{2} \int_{\text {brane }} d^{4} x \sqrt{|g|} R .
$$

This term is the usual 4D Einstein-Hilbert term computed here on the brane and with the induced metric, with $M_{\mathrm{Pl}}$ a mass parameter. The latter can be interpreted as the usual 4D reduced Planck mass, from the calculation (see below) of the force between two static massive sources on a flat brane and bulk background. ${ }^{2}$ The origin of $S_{e h}$ in brane world models is discussed in more detail in [6-8]. It arises generically from quantum correction coming from the coupling between bulk space-time and brane localized matter fields when the conformal invariance of the brane theory is broken (see, e.g., [14]). In the model at hand the dynamics of gravity is then governed by the sum of the two kinetic terms $S_{E H}$ and $S_{e h}$.

As a consequence of the presence of the brane-induced term (3), one can show [6] that the gravitational force experienced by two static pointlike sources located on the brane is the usual $4 \mathrm{D}$ gravitational $1 / r^{2}$ force for distances smaller than the crossover scale $r_{c}$ defined by

$$
r_{c}=\frac{M_{\mathrm{Pl}}^{2}}{2 M_{(5)}^{3}} .
$$

For distances larger than $r_{c}$, on the other hand, the force turns to a $5 \mathrm{D}$ regime where it follows the $5 \mathrm{D} 1 / r^{3}$ behavior. On scales smaller than $M_{(5)}^{-1}$ one also expects modifications in the gravity law; however, for the parameter choice relevant to this work, the modifications occur on scales much smaller than those accessible by gravity experiments [9].

This perturbative behavior has an exact parallel in cosmology, where one can show [4] that, for a $Z_{2}$ symmetric brane world (see $[15,17,18]$ for discussions of cases where the $Z_{2}$ symmetry is relaxed), the expansion of the Universe is governed by the usual 4D Friedmann's equations whenever

\footnotetext{
${ }^{1}$ In the following, we use upper case latin letters $A, B, \ldots$ to denote $5 \mathrm{D}$ indices, greek letters $\mu, \nu, \ldots$ to denote indices parallel to the brane world volume, 5 an index transverse to the brane, and latin letters $i, j, \ldots$ to denote space-like indices parallel to the brane world volume.

${ }^{2}$ We will not address here the issue of the van Dam-VeltmanZakharov (vDVZ) discontinuity, see [11] and [12,13] for discussions of this issue.
}

the Hubble radius $H^{-1}$ is smaller than $r_{c}$, and enters into a nonconventional regime for larger Hubble radii.

In the following section we will discuss in greater detail this cosmological evolution. At this point let us first say that an obvious criteria that the model should reach in order to comply with the known behavior of gravity at large distance, as well as with the observed cosmology, is that $r_{c}$ should be made large enough. The more stringent limit comes indeed from cosmology requiring $r_{c}$ to be of the order of, or larger than, today's Hubble radius $H_{0}^{-1}$. When $r_{c} \sim H_{0}^{-1}$, one thus expects that cosmology is very close to standard cosmology up to very late time, and in particular all successes of standard cosmology such as big bang nucleosynthesis (BBN) are left unchanged by this choice of parameters. However, the very recent evolution of the universe is different. Indeed, as will be noted in more detail in the next section, a particular class of solutions shows a late time accelerated expansion without the need for a nonzero cosmological constant. For values of $r_{c}$ of order $H_{0}^{-1}$, as needed to fit the Supernovae observations (see Sec. III A), one finds from Eq. (4) that $M_{(5)} \sim 10-100 \mathrm{MeV}$. Such a low value of the 5D Planck mass is perfectly consistent with observations and high energy experiments as shown in $[8,9]$. Induced-gravity models have been shown to provide a framework for realizing models with a very low quantum gravity scale without conflicting with any experimental facts [9].

\section{B. Background cosmological dynamics}

In the model considered here, the geometry of our 4D universe is at all time described by an ordinary FriedmannLemaitre-Robertson-Walker (FLRW) space-time with a line element of the form

$$
\begin{aligned}
d s^{2} & =g_{\mu \nu} d x^{\mu} d x^{\nu} \\
& =-d t^{2}+a^{2}(t) d x^{i} d x^{j} \gamma_{i j} \\
& =-d t^{2}+a^{2}(t)\left[d r^{2}+S_{k}^{2}(r) d \psi^{2}\right],
\end{aligned}
$$

where $\psi$ are angular coordinates, $k=-1,0,1$ parametrizes the brane world spatial curvature, and $S_{k}$ is given by

$$
S_{k}(r)= \begin{cases}\sin r & (k=1) \\ \sinh r & (k=-1) \\ r & (k=0) .\end{cases}
$$

The cosmological standard observers are assumed, as usual, to be at rest with respect to the comoving coordinates $x^{i}$. The only difference with standard cosmology is in the dynamics of the metric which is encoded into Friedmann-like equations different from the ordinary $4 \mathrm{D}$ ones. For a given content of the universe, with total energy density $\rho$ (and pressure $p$ ), the standard first Friedmann's equation is now replaced by $[4]$

$$
H^{2}+\frac{k}{a^{2}}=\left(\sqrt{\frac{\rho}{3 M_{\mathrm{Pl}}^{2}}+\frac{1}{4 r_{c}^{2}}}+\frac{1}{2 r_{c}}\right)^{2},
$$

where 


$$
H \equiv \frac{1}{a} \frac{d a}{d t}
$$

is the Hubble parameter of our universe. ${ }^{3}$ The energymomentum conservation equation, on the other hand, takes the usual form

$$
\dot{\rho}+3 H(p+\rho)=0 .
$$

Equations (9) and (11) are all what is needed to characterize the cosmology we are interested in here. They lead to

$$
\begin{aligned}
H^{2}(z)= & H_{0}^{2}\left\{\Omega_{k}(1+z)^{2}\right. \\
& \left.+\left(\sqrt{\Omega_{r_{c}}}+\sqrt{\Omega_{r_{c}}+\sum_{\alpha} \Omega_{\alpha}(1+z)^{3\left(1+w_{\alpha}\right)}}\right)^{2}\right\},
\end{aligned}
$$

where $z$ is the redshift and we have assumed that $\rho$ is given by the sum of the energy densities $\rho_{\alpha}$ of different components (labeled by $\alpha$ ) with constant equation of state parameters $w_{\alpha}$. The $\Omega$ s for matter and curvature are defined in the usual way by

$$
\begin{aligned}
& \Omega_{\alpha} \equiv \frac{\rho_{\alpha}^{0}}{3 M_{\mathrm{Pl}}^{2} H_{0}^{2} a_{0}^{3\left(1+w_{\alpha}\right)}}, \\
& \Omega_{k} \equiv \frac{-k}{H_{0}^{2} a_{0}^{2}},
\end{aligned}
$$

whereas $\Omega_{r_{c}}$ is given by

$$
\Omega_{r_{c}} \equiv \frac{1}{4 r_{c}^{2} H_{0}^{2}} .
$$

The normalization condition for the $\Omega \mathrm{s}$,

$$
\Omega_{k}+\left(\sqrt{\Omega_{r_{c}}}+\sqrt{\Omega_{r_{c}}+\sum_{\alpha} \Omega_{\alpha}}\right)^{2}=1,
$$

differs from the usual relation $\Omega_{k}+\Sigma_{\alpha} \Omega_{\alpha}=1$.

Equation (9) implies that whenever $\rho / M_{\mathrm{Pl}}^{2}$ is large compared to $1 / r_{c}^{2}$ (or in other words, whenever $H^{-1}$ is small with respect to $r_{c}$ ), the cosmological evolution follows that of standard cosmology. In this case Eq. (9) reduces to the standard Friedmann's equation

$$
H^{2}+\frac{k}{a^{2}}=\frac{\rho}{3 M_{\mathrm{Pl}}^{2}} .
$$

When (and if) $\rho$ is driven to smaller values by the cosmic expansion, the expansion of the Universe enters into a nonconventional phase and asymptotes to a de Sitter solution when $\rho$ becomes negligible with respect to $M_{\mathrm{Pl}}^{2} / r_{c}^{2}$. One has a transition to an accelerated expansion happening approximately when the Hubble radius $H^{-1}$ crosses the threshold $r_{c}$. We would like to stress that this last accelerated phase is not triggered by a cosmological constant (that can be consistently set to zero) but is due to the presence of two kinetic terms for the graviton in the action. Namely, bulk gravity sees the induced kinetic term on the brane (3) as a source term, and for an empty universe, there is a self-inflationary solution $^{4}$ to Einstein's equations to which a universe with decreasing energy will asymptote. This solution acts as a late time attractor to early standard cosmology.

In the following we will then only consider a universe with a zero cosmological constant, and usual (dark, baryonic, ...) matter content. One can further notice that the above described cosmology is also exactly reproduced by standard cosmology with a dark energy component with a $z$-dependent equation of state parameter $w_{X}^{e f f}(z)$. For a universe containing only nonrelativistic matter, $w_{X}^{\text {eff }}(z)$ is given by (see [5])

$$
w_{X}^{e f f}(z)=\frac{1}{\left(\sqrt{\frac{4 \Omega_{r_{c}}}{\Omega_{M}(1+z)^{3}}+4}\right)\left(\sqrt{\frac{\Omega_{r_{c}}}{\Omega_{M}(1+z)^{3}}}+\sqrt{\frac{\Omega_{r_{c}}}{\Omega_{M}(1+z)^{3}}+1}\right)}-1 .
$$

\footnotetext{
${ }^{3}$ There is another set of solutions for a $Z_{2}$ symmetrical brane. Those were derived in [4] and are not considered here.

${ }^{4}$ This solution is in a way the late time analog of Starobinsky's first model of inflation where terms quadratic in the Ricci tensor are sourcing similarly a self-inflationary solution [16].
} 
At large redshift $w_{X}^{\text {eff }}$ tends toward $-1 / 2$ reflecting the fact that the dominant term in Eq. (12), after matter and curvature terms, redshift as $(1+z)^{3 / 2}$ at large $z$. At low $z$, however, $w_{X}^{e f f}$ decreases toward an $\left(\Omega_{k}, \Omega_{M}\right)$-dependent asymptotic value. For a flat universe, the latter is simply given by ${ }^{5}$ $-1 /\left(1+\Omega_{M}\right)$.

In the following sections we give the results of fitting SNIa and CMB observables with different cosmological parameters in the framework of the cosmology defined by Eqs. (9) and (11). We will denote $\boldsymbol{\theta}$ a set of cosmological parameters such as $\Omega_{r_{c}}$ or $\Omega_{M}$ characterizing a given cosmology.

\section{CONFRONTATION WITH OBSERVATIONS}

\section{A. Confrontation with supernovae observations}

We have fitted the supernovae data set from the SCP [2] with the luminosity distance calculated using Eq. (12). Because the geometry of the Universe is given by usual FLRW (5) one can use the standard formula for the luminosity distance $d_{L}$ as a function of the redshift $z$,

$$
d_{L}=H_{0}^{-1}(1+z) \frac{S_{k}\left[\sqrt{\left|\Omega_{k}\right|} d_{C}(z)\right]}{\sqrt{\left|\Omega_{k}\right|}},
$$

with $d_{C}(z)$ defined by

$$
d_{C}(z)=\int_{0}^{z} H_{0} \frac{d y}{H(y)}
$$

and $H(z)$ given by Eq. (12). We then use this luminosity distance to fit the data. The fit is done using 4 free parameters: the cosmological parameters $\boldsymbol{\theta}=\left(\Omega_{M}, \Omega_{r_{c}}\right)$, the intrinsic magnitude of the supernova $\mathcal{M}$, and a parameter $\alpha$ related to the intrinsic luminosity-decline rate relation (stretch factor $s) \cdot \chi^{2}$ is given by

$$
\begin{aligned}
& \chi^{2}(\boldsymbol{\theta}, \alpha, \mathcal{M}) \\
& \quad=\sum_{i=1}^{n} \frac{\left\{\mathcal{M}+\alpha\left(1-s_{i}\right)+5 \log _{10}\left[d_{L}\left(\boldsymbol{\theta}, z_{i}\right)\right]-m_{i}\right\}^{2}}{\sigma_{i}^{2}} .
\end{aligned}
$$

The data set consisting of 54 supernovae (18 nearby ones and 36 at high redshift) is shown in Fig. 1. Since we assume no prior knowledge of the parameters and as we are not interested by $\alpha$ and $\mathcal{M}$, we have to marginalize over them. We do this in a Bayesian framework assuming flat priors and Gaussian errors. These integrations can be carried out analytically, as shown in [19]. We quote the results in Appendix A. We have then computed confidence contours for the models $\boldsymbol{\theta}=\left(\Omega_{M}, \Omega_{r_{c}}\right)$ model with no prior on the cosmology. These contours are plotted in Fig. 2.

\footnotetext{
${ }^{5}$ E.g., for $\Omega_{M}=0.3$ and $k=0, w_{X}^{e f f}$ at low $z$ tends toward -0.77 .
}

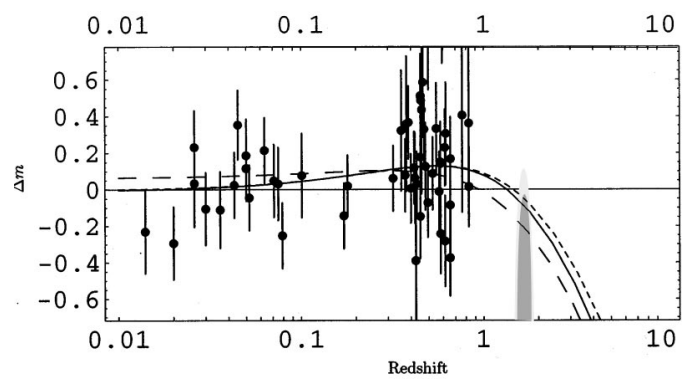

FIG. 1. Magnitude vs redshift diagram for the SNIa data of Ref. [2] used in this paper. All magnitude are plotted, respectively, to an empty universe $\left(\Omega_{M}=0\right.$ and $\left.\Omega_{\Lambda}=0\right)$. Over-plotted are three different flat cosmological models: the best fit flat model in standard cosmology (with $\Omega_{M}=0.28$ and $\Omega_{\Lambda}=0.72$, solid line), in the gravitational leakage cosmology (with $\Omega_{M}=0.18$, dotted line) and a flat model in the gravitational leakage cosmology with $\Omega_{M}=0.3$ (dashed line). We also show two approximate confidence level intervals for the $z=1.7$ supernova of Ref. [3]; the outer light-gray surface represents the $95 \%$ confidence intervals and the inner dark gray surface represents approximately the $68 \%$ confidence interval. This last supernova was not included in the fit. The values of $\alpha$ (related to the stretch factor) and $\mathcal{M}$ (intrinsic magnitude) have been fitted independently for all the models. The data are plotted here with $\alpha=0.6$.

Assuming a spatially flat space-time, one is only left with one free parameter (after integration over $\mathcal{M}$ and $\alpha$ ), e.g. $\Omega_{M} \cdot \Omega_{r_{c}}$ is then given by the normalization condition (16)

$$
\Omega_{r_{c}}=\left(\frac{1-\Omega_{M}}{2}\right)^{2}, \quad \Omega_{r_{c}}<1 \quad \text { and } \quad \Omega_{M}<1
$$

The results of the $\chi^{2}$ minimization gives for a flat universe (one sigma levels)

$$
\Omega_{M}=0.18_{-0.06}^{+0.07} \quad \text { or } \quad \Omega_{r_{c}}=0.17_{-0.02}^{+0.03}
$$

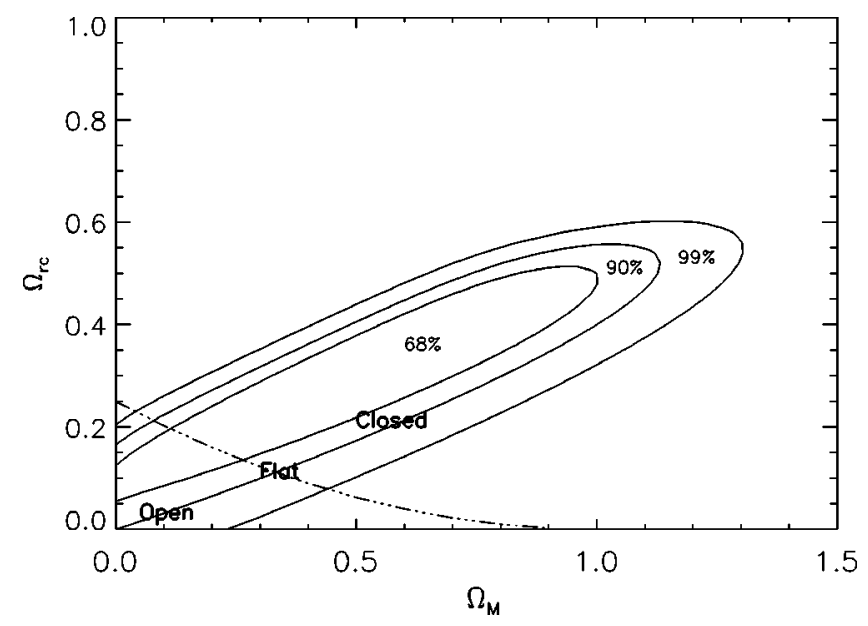

FIG. 2. $68.3 \%, 90 \%$ and $99 \%$ confidence regions for $\left(\Omega_{M}, \Omega_{r_{c}}\right)$ in the gravitational leakage scenario, assuming no prior knowledge of $\alpha$ and $\mathcal{M}$. 
with $\chi^{2}=57.96$ for 52 (54 SNe, 2 parameters) degrees of freedom. ${ }^{6}$ This best fit model is shown in Fig. 1. Equation (23) leads to an estimate for $r_{c}$ in terms of the Hubble radius $H_{0}^{-1}$ given by

$$
r_{c}=1.21_{-0.09}^{+0.09} H_{0}^{-1}
$$

\section{B. Confrontation with CMB observations}

Another set of cosmological observables which has recently been measured with great precision is the CMB temperature power spectrum. In this section we would like to compare the predictions of the model considered in this paper to the results of these observations.

For this purpose, we used a modified CMBFAST [22] replacing the first Friedmann's equation by Eq. (9). The equations for the growth of cosmological perturbations were kept the same as in usual cosmology (except for the background evolution). As is discussed qualitatively in Appendix B, this is justified for the small scale perturbations and for processes happening early enough in the history of the Universe. On the other hand, one can expect deviations from the standard picture at large scale (and late time) where (and when) the effect of the extra dimension began to be felt. This concerns scales of order of today's Hubble radius and processes happening in the late history of the Universe. A more refined discussion of this, which involves the integration of bulk equations of motions for perturbations, is left for future work [23].

We explored the six-dimensional parameter space, $\boldsymbol{\theta}$ $=\left(\Omega_{k}, \Omega_{r_{c}}, \omega_{\mathrm{d}}, \omega_{\mathrm{b}}, n, A\right)$, where $\omega_{\mathrm{d}}=\Omega_{\mathrm{cdm}} h^{2}, \omega_{\mathrm{b}}=\Omega_{\mathrm{b}} h^{2}$ and $A$ and $n$ are the amplitude and slope of the primordial spectrum of perturbations. We used a Markov chain method to explore the likelihood in this parameter space. When it has converged the method produces a chain of models that are sampled from the probability distribution of $\boldsymbol{\theta}$. The details of our procedure are given in Appendix C.

Figure 3 shows the probability distribution for each of the six parameters obtained. As expected the CMB data prefers spatially flat models. Figure 4 shows the results of our analysis in the $\Omega_{M^{-}} \Omega_{r_{c}}$ plane. The shaded region was drawn to contain approximately $95 \%$ of the models in our chain, the line marks the location of spatially flat models. The constraint on $\Omega_{r_{c}}$ is coming mainly from the position of the acoustic peaks so there is a natural degeneracy in the $\Omega_{M^{-}} \Omega_{r_{c}}$ plane which is apparent in the plot.

The probability distribution for $\Omega_{k}$ shown in Fig. 3 peaks around $\Omega_{k}=0$, a spatially flat universe. Thus it is natural to further restrict ourselves to flat universes which we can do by

\footnotetext{
${ }^{6}$ These numerical results are in agreement with the fit done in [20]. We, however, disagree with the conclusions of that work as will be discussed later (see also Ref. [21] for a discussion of this paper). Note in particular that, contrary to the claims made in [20], the $z=1.7$ supernova of Ref. [3] is fitted as well by the model considered in this paper or by standard cosmology with a cosmological constant (see Fig. 1).
}
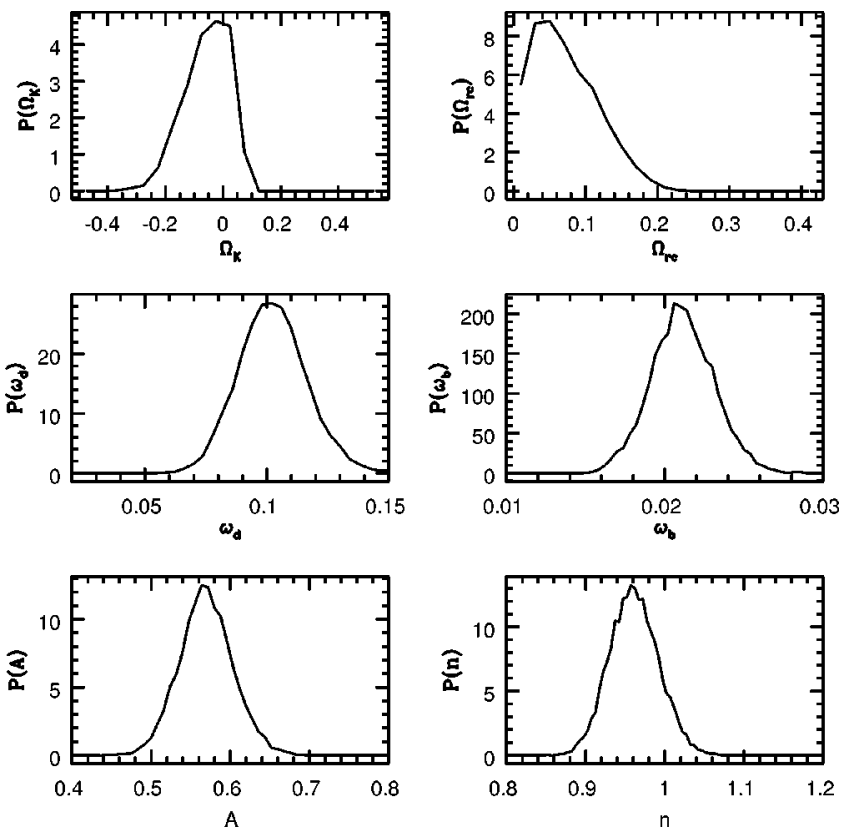

FIG. 3. Marginal distribution for each of the 6 parameters used.

considering only samples in our chain with negligible curvature. The probability distribution for $\Omega_{M}$ under this assumption is shown in Fig. 5.

Figure 5 shows that a model with $\Omega_{M}=0.3$ provides a good fit to both SN and CMB data. It should be noted, however, that the CMB prefers a slightly larger value of $\Omega_{M}$ than the $\mathrm{SN}$, although both ranges overlap at $1 \sigma$. In turn, the $\mathrm{CMB}$ can constrain the physical densities in matter quite accurately, $\omega_{\mathrm{M}}+\omega_{\mathrm{b}} \approx 0.12$. This constraint translates $\Omega_{M}$ $=0.3$ to a Hubble constant $h \approx 0.63$ in good agreement with

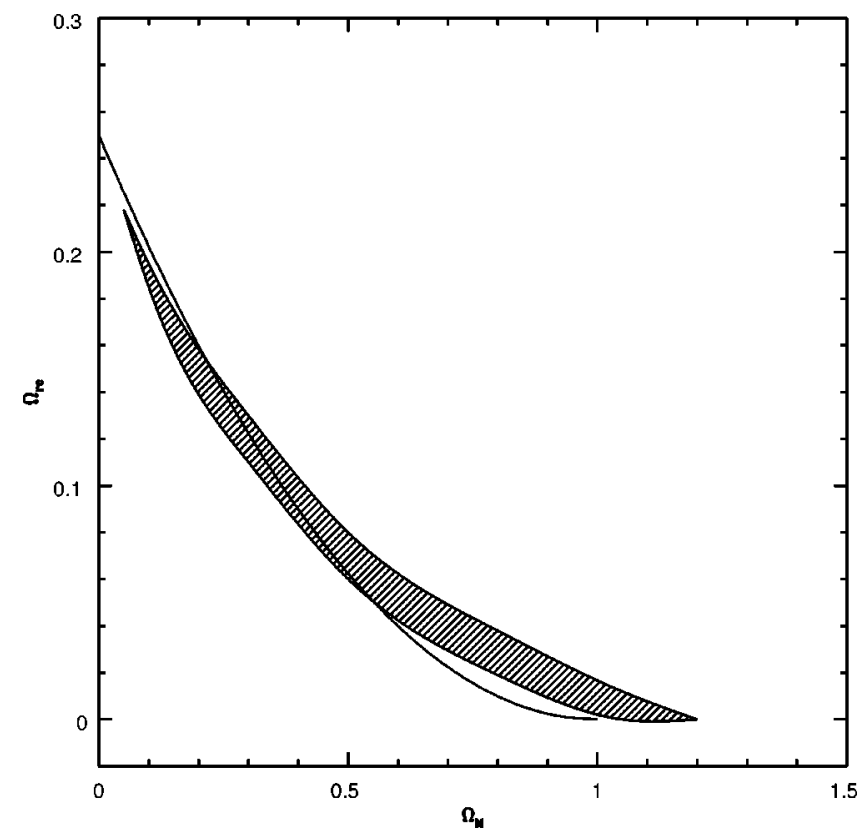

FIG. 4. Allowed region in the $\Omega_{M^{-}} \Omega_{r_{c}}$ plane (shaded). The line shows the location of spatially flat models. The shaded region was drawn to contain approximately $95 \%$ of the models in our chain. 


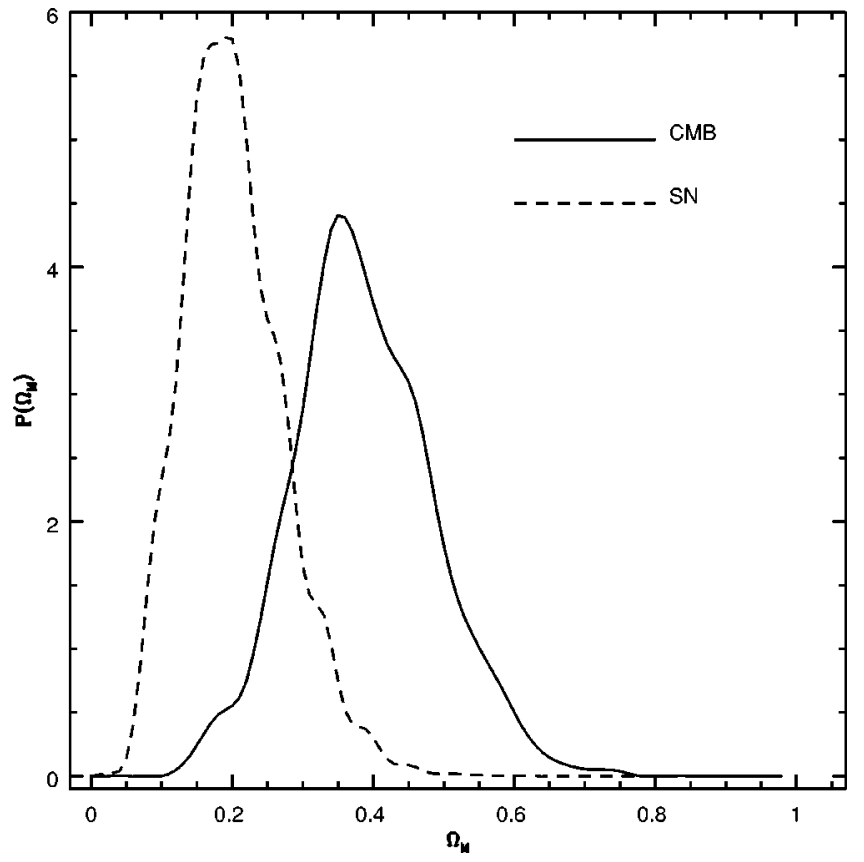

FIG. 5. Marginal distributions for $\Omega_{M}$ under the assumption that the universe is spatially flat. The solid line shows the results from $\mathrm{CMB}$ and the dashed line from the SN.

direct measurements, e.g., $h=0.72 \pm 0.08$ from the HST key project [40].

In Fig. 6 we show what we could call our "concordance" model, $\boldsymbol{\theta}=\left(\Omega_{k}, \Omega_{r_{c}}, \omega_{\mathrm{d}}, \omega_{\mathrm{b}}, n, A\right)=(0,0.1225,0.1,0.02,0.96$, 0.57 ) which has $\Omega_{M}=0.3$ and $\chi^{2} \approx 140$ for the full data set

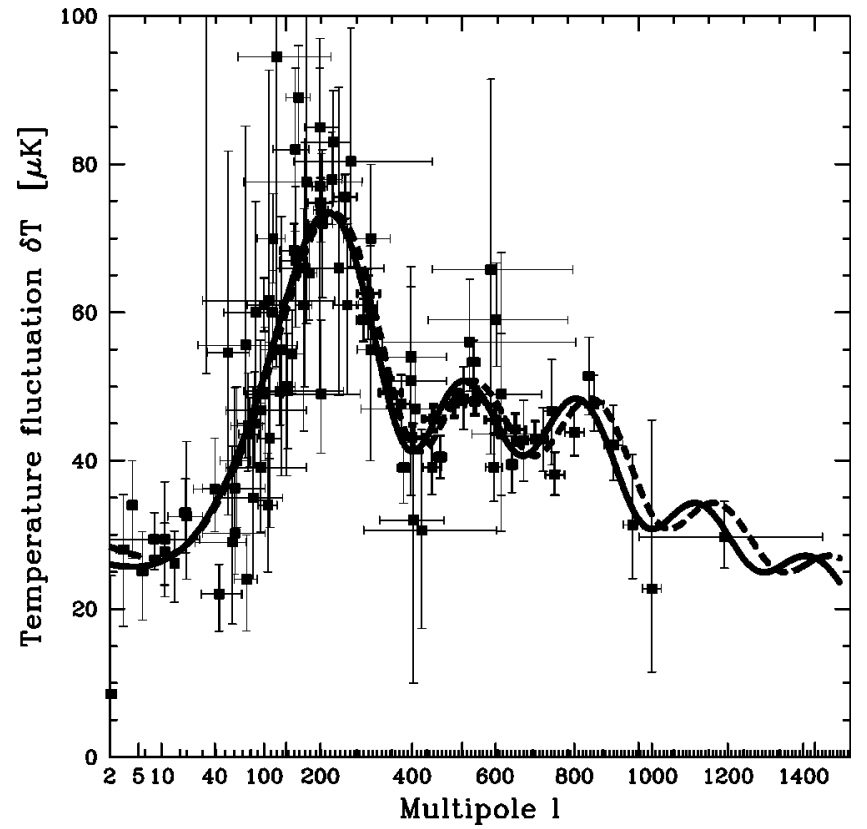

FIG. 6. Model predictions and current $\mathrm{CMB}$ data. The solid curve curve is for a model with $\boldsymbol{\theta}=\left(\Omega_{k}, \Omega_{r_{c}}, \omega_{\mathrm{d}}, \omega_{\mathrm{b}}, n, A\right)$ $=(0,0.1225,0.1,0.02,0.96,0.57)$ while the dashed curve is for standard cosmology with a cosmological constant $\Omega_{\Lambda}=0.7$ (other parameters were kept the same).
(135 data points). For reference we also show the power spectra for a standard cosmology model with $\Omega_{\Lambda}=0.7$, a model that has all other parameters the same but where the acceleration is produced by a cosmological constant. Both models provide an acceptable fit to the current data, but because their angular diameter distance to the last scattering surface differs by approximately $4 \%$ they should be easily distinguishable by future generation of $\mathrm{CMB}$ experiments. The difference at low multipoles should be regarded with care because on this very large scale the physical effects we ignored could be relevant (see Appendix B).

\section{CONCLUSIONS}

The fits done in this work show that the model of an accelerated universe through gravitational leakage into an extra dimension of Refs. $[4,5]$ is in current agreement with SNIa and CMB data. The degeneracies in parameter estimations using one data set (e.g. CMB) can be partially lifted using the other (e.g. SNIa) as in standard cosmology. The supernovae data prefer a slightly lower value of $\Omega_{M}\left(\Omega_{M}\right.$ $\left.=0.18_{-0.06}^{+0.07}\right)$ than the CMB for a flat universe; however, a concordance model with $\left(\Omega_{k}, \Omega_{r_{c}}, \omega_{\mathrm{d}}, \quad \omega_{\mathrm{b}}, n, A\right)$ $=(0,0.1225,0.1,0.02,0.96,0.57)$ which has $\Omega_{M}=0.3$ [and $\chi^{2} \approx 140$ for the full data set (135 data points)] provide a good fit to both sets, all the more as we have not included systematic errors in our parameter estimations. For this model the crossover distance between $4 \mathrm{D}$ and $5 \mathrm{D}$ gravity is given by $r_{c} \sim 1.4 H_{0}^{-1}$.

We have also given the equation of evolution for cosmological perturbations. Those equations were used to justify the approximation we made to compute cosmological perturbations, namely we used standard four dimensional evolution equations over a background with a scale factor given by the accelerated solution given in [5]. This is justified for small scale CMB anistotropies (scale smaller than the crossover scale $r_{c}$ ). From those equations, and the known behavior of gravity in the model at hand, one can also expect modifications in the growth of large scale structure. This could potentially lead to a way to discriminate between standard cosmology and the model considered in this work, and is left for future investigation.

We want to end by noting that the model under consideration is very predictive in the sense that future observations have the potential to rule it out. In contrast to quintessence models, this model has the same number of free parameters as the usual LCDM model. With the advent of new precision cosmological measurements such as new SNIa observations, CMB measurements, ongoing galaxy surveys such as Sloan and $2 \mathrm{dF}$, weak lensing surveys, etc., it should be possible to test the model very accurately (for a recent summary of how different observations will constrain the matter content of the universe see [41] and references therein).

\section{ACKNOWLEDGMENTS}

We thank Gia Dvali, Andrei Gruzinov, Arthur Lue, and Roman Scoccimarro for useful discussions. The work of C.D. is sponsored in part by NSF Award PHY 9803174, and 
by David and Lucile Packard Foundation through Grant No. 99-1462. M.Z. is supported by the David and Lucile Packard Foundation and NSF grants AST-0098506 and PHY0116590 .

\section{APPENDIX A: MARGINALIZATION FOR SUPERNOVAE}

Following [19] the $\chi^{2}$ defined by Eq. (21) can be integrated analytically over $\mathcal{M}$ and $\alpha$ to yield

$$
\begin{aligned}
\chi_{\alpha \text {-int }}^{2}(\boldsymbol{\theta}) & =-2 \ln \left[\int_{-\infty}^{\infty} d \alpha \exp \left(-\frac{1}{2} \chi_{\mathcal{M} \text {-int }}^{2}(\boldsymbol{\theta}, \alpha)\right)\right] \\
& =A^{\prime}-\frac{B^{\prime 2}}{C^{\prime}}-\frac{\left(F-\frac{B^{\prime} E}{C^{\prime}}\right)^{2}}{D-\frac{E^{2}}{C^{\prime}}}, \\
A^{\prime} & =\sum_{i=1}^{n} \frac{\left\{5 \log _{10}\left[d_{L}\left(\boldsymbol{\theta}, z_{i}\right)\right]-m_{i}\right\}^{2}}{\sigma_{i}^{2}}, \\
B^{\prime} & =\sum_{i=1}^{n} \frac{5 \log _{10}\left[d_{L}\left(\boldsymbol{\theta}, z_{i}\right)\right]-m_{i}}{\sigma_{i}^{2}}, \\
C^{\prime} & =\sum_{i=1}^{n} \frac{1}{\sigma_{i}^{2}}, \\
F^{2} & =\sum_{i=1}^{n} \frac{\left(1-s_{i}\right)^{2}}{\alpha_{i}^{2}}, \\
E & =\sum_{i=1}^{n} \frac{\left(1-s_{i}\right)}{\alpha_{i}^{2}}, \\
\sigma_{i}^{2} & \left\{5 \log _{10}\left[d_{L}\left(\boldsymbol{\theta}, z_{i}\right)\right]-m_{i}\right\}\left(1-s_{i}\right) \\
&
\end{aligned}
$$

where $\chi_{\mathcal{M} \text {-int }}^{2}(\boldsymbol{\theta}, \alpha)$ is defined by

$$
\chi_{\mathcal{M} \text {-int }}^{2}(\boldsymbol{\theta}, \alpha)=-2 \ln \left[\int_{-\infty}^{\infty} d \mathcal{M} \exp \left(-\frac{1}{2} \chi^{2}(\boldsymbol{\theta}, \mathcal{M}, \alpha)\right)\right]
$$

\section{APPENDIX B: DYNAMICS OF SCALAR COSMOLOGICAL PERTURBATIONS}

We briefly summarize here the equations governing the cosmological perturbations in the model at hand. These equations will be derived and discussed in more details elsewhere, and are only given here for the case of a flat universe.

Our starting point is an equation derived in [24], relating the 4D Einstein's tensor $G_{\mu \nu}$ to a tensor $\Pi_{\mu \nu}$ quadratic in whatever source $\widetilde{T}_{\mu \nu}$ of 5D Einstein's equations is localized on the brane, and a traceless tensor $\mathcal{E}_{\mu \nu}$ defined in terms of the $5 \mathrm{D}$ bulk Weyl tensor. The corresponding equation reads

$$
G_{\mu \nu}=\frac{1}{M_{(5)}^{6}} \Pi_{\mu \nu}-\mathcal{E}_{\mu \nu}
$$

with $\Pi_{\mu \nu}$ given by

$$
\begin{aligned}
\Pi_{\mu \nu}= & -\frac{1}{4} \widetilde{T}_{\mu \alpha} \widetilde{T}_{\nu}^{\alpha}+\frac{1}{12} \widetilde{T} \widetilde{T}_{\mu \nu}+\frac{1}{8} \widetilde{T}_{\alpha \beta} \widetilde{T}^{\alpha \beta} g_{\mu \nu} \\
& -\frac{1}{24} \widetilde{T}^{2} g_{\mu \nu},
\end{aligned}
$$

and $\mathcal{E}_{\mu \nu}$ is defined by

$$
\mathcal{E}_{\mu \nu}=C_{\mu 5 \nu}^{5},
$$

from the bulk Weyl's tensor ${ }^{7} C_{B C D}^{A}$. In our case $\widetilde{T}_{\mu \nu}$ is given by

$$
\widetilde{T}_{\mu \nu}=T_{\mu \nu}-M_{\mathrm{Pl}}^{2} G_{\mu \nu},
$$

where $T_{\mu \nu}$ is the brane energy momentum tensor and $G_{\mu \nu}$ is the 4D Einstein's tensor. $T_{\mu \nu}$ is conserved with respect to the $4 \mathrm{D}$ metric on the brane, so that one has

$$
\begin{aligned}
& D_{\mu} T_{\nu}^{\mu}=0, \\
& D_{\mu} \widetilde{T}_{\nu}^{\mu}=0,
\end{aligned}
$$

where $D_{\mu}$ denotes the covariant derivative compatible with the $4 \mathrm{D}$ metric on the brane, and the last equality follows from Bianchi identities. Equations (B1) and (B5) lead to the background equation of motion (12), once one knows the background expression for $\mathcal{E}_{\mu \nu}$. In the cosmological case, $\mathcal{E}_{\mu \nu}$ is in general given by some version of Birkhoff's theorem [25-29]. We have assumed for simplicity in Eq. (12) that it vanishes in the background, in which case the five dimensional space-time is simply a Minkowski space-time. ${ }^{8}$

We now derive from Eq. (B1) the evolution equations for the cosmological perturbations. We write

$$
\begin{aligned}
G_{\nu}^{\mu} & ={ }^{B} G_{\nu}^{\mu}+\delta G_{\nu}^{\mu}, \\
T^{\mu} & ={ }^{B} T_{\nu}^{\mu}+\delta T_{\nu}^{\mu}, \\
\mathcal{E}_{\nu}^{\mu} & =\delta \mathcal{E}^{\mu}{ }_{\nu},
\end{aligned}
$$

where the superscript $B$ stands for the background value of the corresponding tensor component. We define then the sca-

\footnotetext{
${ }^{7}$ We have chosen here implicitly a Gaussian normal coordinate with respect to the brane.

${ }^{8} \mathrm{As}$ far as the background is concerned a nonvanishing $\mathcal{E}_{\mu \nu}$ is manifesting itself as a radiation component into the Friedmann's equations; see Ref. [4] where the background equations are given in full generality.
} 
lar perturbations in energy density $\delta \rho$, momentum $\delta q$, pressure $\delta P$, and anisotropic stress $\delta \pi$ for ordinary matter as

$$
\begin{aligned}
& \delta T_{0}^{0}=-\delta \rho, \\
& \delta T_{i}^{0}=\nabla_{i} \delta q, \\
& \delta T_{j}^{i}=\delta P \delta_{j}^{i}+\left(\nabla^{i} \nabla_{j}-\frac{1}{3} \delta_{j}^{i} \nabla^{2}\right) \delta \pi,
\end{aligned}
$$

where $\nabla_{i}$ is the covariant derivative adapted to the background spatial metric $\gamma_{i j}$ parallel to the brane. We also define similar quantities for the Weyl's fluid, following [30-33]

$$
\begin{gathered}
\delta \mathcal{E}_{0}^{0}=\frac{1}{M_{\mathrm{Pl}}^{2}} \delta \rho_{\mathcal{E}} \\
\delta \mathcal{E}_{i}^{0}=-\frac{1}{M_{\mathrm{Pl}}^{2}} \nabla_{i} \delta q_{\mathcal{E}} \\
\delta \mathcal{E}_{j}^{i}=-\frac{1}{M_{\mathrm{Pl}}^{2}}\left[\delta P_{\mathcal{E}} \delta_{j}^{i}+\left(\nabla^{i} \nabla_{j}-\frac{1}{3} \delta_{j}^{i} \nabla^{2}\right) \delta \pi_{\mathcal{E}}\right]
\end{gathered}
$$

The Weyl's fluid is related to the perturbation of the bulk Weyl's tensor (gravitational waves in the bulk) through Eq. (B3).

Other useful quantities are the trace, $\delta G_{T}$, and traceless traceless part, $\delta G_{T F}$, of $\delta G_{j}^{i}$ defined by

$$
\delta G_{j}^{i}=\delta G_{T} \delta_{j}^{i}+\left(\nabla^{i} \nabla_{j}-\frac{1}{3} \delta_{j}^{i} \nabla^{2}\right) \delta G_{T F} .
$$

After some algebra, one gets then from Eq. (B1) the perturbed Einstein's tensors over the background (11), (12)

$$
\begin{array}{r}
\delta G_{0}^{0}\left(1-\frac{1}{2 H r_{c}}\right)=-\frac{1}{M_{\mathrm{Pl}}^{2}}\left(\delta \rho-\frac{\delta \rho_{\mathcal{E}}}{2 H r_{c}}\right), \quad \text { (B17) } \\
\delta G_{i}^{0}\left(1-\frac{1}{2 H r_{c}}\right)=\frac{1}{M_{\mathrm{Pl}}^{2}}\left(\nabla_{i} \delta q-\frac{1}{2 H r_{c}} \nabla_{i} \delta q_{\mathcal{E}}\right), \quad(\mathrm{B} 18) \\
\delta G_{T F}\left(1-\frac{H}{r_{c}\left(\dot{H}+2 H^{2}\right)}\right)=\frac{1}{M_{\mathrm{Pl}}^{2}}\left(\delta \pi-\delta \pi_{\mathcal{E}} \frac{H}{r_{c}\left(\dot{H}+2 H^{2}\right)}\right), \\
\delta G_{T}\left(1-\frac{1}{2 H r_{c}}\right)=\frac{1}{M_{\mathrm{Pl}}^{2}}\left(\delta P-\frac{1}{2 H r_{c}} \delta P_{\mathcal{E}}+\frac{\dot{H}}{3 H^{2}} \frac{\delta \rho-\delta \rho_{\mathcal{E}}}{2 H r_{c}-1}\right) .
\end{array}
$$

These equations replace the perturbed Einstein's equations of ordinary cosmology (see, e.g. [34] for a review).

One can derive from Eq. (B5) the usual conservation equations for the matter perturbations. As far as the Weyl's fluid is concerned, by taking the covariant derivative of Eq. (B1) and using Eq. (B5) one can show that the Weyl's fluid energy density $\delta \rho_{\mathcal{E}}$ is conserved but that the Weyl's fluid momentum $\delta q_{\mathcal{E}}$ in general is not [31]. Moreover, one does not have an evolution equation for the Weyl's fluid anisotropic stress $\delta \pi_{\mathcal{E}}$. This means that the system of equations for cosmological perturbations does not close on the brane, and one needs to solve the equations of motion for gravitational waves in the bulk (see [31]). On large scales, however, the usual adiabatic curvature perturbation on hypersurfaces of uniform (ordinary or Weyl) matter density is conserved [31], since it is a mere consequence of the conservation of the energy density perturbation [35]. However, one still cannot compute the Sachs-Wolf effect because of the lack of knowledge of $\delta \pi_{\mathcal{E}}$ [31].

Let us make here some simple remarks. In the formalism used so far, the deviation from usual 4D cosmological perturbations equations can be separated into two different parts. We first note that the direct coupling between ordinary matter and gravitational perturbations is $H$ dependent, for example one can rewrite Eq. (B17) as

$$
\delta G_{0}^{0}=-\frac{1}{\widetilde{M}_{\mathrm{Pl}}^{2}}\left(\delta \rho-\frac{\delta \rho_{\mathcal{E}}}{2 H r_{c}}\right)
$$

with the effective direct gravitational coupling between matter and gravity given by

$$
\tilde{M}_{\mathrm{Pl}}^{2}=M_{\mathrm{Pl}}^{2}\left(1-\frac{1}{2 H r_{c}}\right)
$$

One can check that this coupling is never negative for the late time accelerated solution considered in this paper, since one always has $H r_{c} \geqslant 1$. Moreover, in the early time of the Universe (whenever $H r_{c} \gg 1$ ), one has $\tilde{M}_{\mathrm{Pl}} \sim M_{\mathrm{Pl}}$ so that one can consistently ignore this effect at least up to last scattering (in contrast to what is happening in usual brane cosmology), which is all what matters as far as CMB is concerned. At the epoch of last scattering, for example, $\tilde{M}_{\mathrm{Pl}}$ coincides with $M_{\mathrm{Pl}}$ within a part per thousand. However, $H r_{c}$ becomes of order unity at late time [see Eq. (23)], and one can be concerned that this can have dramatic effects on large scale structure formation. ${ }^{9}$ To be consistent one should also consider in this regime the effects of the Weyl's fluid source terms in the left-hand side of the perturbed Einstein's equations (B17) (B20) (as well as possible nonlinear corrections, see [11]), and this can only be done properly solving for the bulk equations of motions for perturbations. With the formalism used so far, those source terms are the other manifestation of the

\footnotetext{
${ }^{9}$ This could also have a potentially observable signature through standard tests of gravity. We, however, expect that when one looks at fluctuations over a given background, a local curvature scale $l^{-1}$ should typically replace $H^{-1}$ in the above equations so that we do not expect that the $l$ dependence of $\tilde{M}_{\mathrm{Pl}}$ could have observable effects on systems where the curvature is much greater than today's $H_{0}\left(\sim r_{c}^{-1}\right)$. This issue is likely to be related to the disappearance of the vDVZ discontinuity (see [11-13]) and will be discussed elsewhere.
} 
extra dimension that we would like to discuss now qualitatively as far as the CMB is concerned.

We note that those source terms are suppressed with respect to their ordinary matter counterparts by a factor $\mathrm{Hr}_{c}$, in contrast to what happens usually for brane cosmology (see e.g., [33]). This supports the fact that when $\mathrm{Hr}_{c} \rightarrow \infty$ the theory looks more and more four dimensional. One can then start with initial conditions for cosmological perturbations, say after inflation, which are the ones provided by standard 4D cosmology, and set initially all the Weyl's perturbations to zero. The brane perturbations will then feed up nonzero perturbations in the bulk, which will then backreact on the brane through the Weyl's fluid perturbations leading to "gravitational leakage" into the extra dimension. The time scale for this leakage to occur is, however, of order $r_{c}$ which is much larger than the age of the universe at recombination. ${ }^{10}$

This discussion indicates that one can consistently use the usual 4D cosmological perturbation equations for dealing with the growth of small scale fluctuations observed in CMB. The effects of gravitational leakage is then only contained in the background evolution, which affects the growth of the perturbations and also the way they appear on the sky through a different angular diameter distance. We only expect possible deviations on large scale coming from the effects mentioned above, and also possible modifications once compared with large scale structure data. We let these interesting questions be for future investigations, as well as a more careful check of the approximations made here.

\section{APPENDIX C: CMB LIKELIHOOD CALCULATION}

Here we describe the details of the CMB likelihood calculation. To accelerate the calculation of the model predictions we used the $k$-split approximation described in [37]. In

\footnotetext{
${ }^{10}$ This qualitative picture is supported by numerical calculations in a scalar field toy model [36].
}

this approximation the high $l$ power spectra is calculated in a flat model with no dark energy and then shifted appropriately in $l$ using the angular diameter distance to recombination. The likelihood for each model was calculated using the RADPACK $^{11}$ package. We used all currently available CMB data (for a description of the compilation we refer the reader to the RADPACK documentation and to [38]).

For the present study we did not use a grid based method for calculating likelihoods (such as the one described in [37]). Following [39] we instead chose to use the Metropolis-Hastings algorithm to generate a Markov chain of models. Because we are not interested in investigating multiple priors, our parameter space is rather small so we do not need to exploit the CMB degeneracies and we do not want to build a database of models to be used in future studies, the Markov-chain technique was very efficient and extremely easy to implement.

In the Metropolis-Hastings algorithm a chain of models is generated. Models are added to the chain sequentially. To find a new model for the chain values of the parameters are chosen at random (we choose to select models from a Gaussian distribution centered in the last model of the chain with a covariance matrix that is estimated from the chain itself). The likelihood of this new model is compared to the likelihood of the last model in the chain. The new model is always accepted into the chain if the likelihood is larger than that of the last model; if this is not the case it will be accepted with a probability given by the likelihood ratio of the two models. When the chain has converged (i.e., it has run for a sufficiently long time) each model can be taken as an independent sample from the probability distribution $P(\boldsymbol{\theta} \mid \mathbf{d})$, the probability of some particular value of the parameters $(\boldsymbol{\theta})$ given the observed data (d). Once we have the chain of models using histograms we can construct the distribution function of individual parameters.

\footnotetext{
${ }^{11}$ RADPACK is a publicly available software package developed by Lloyd Knox. It can be obtained from http://bubba.ucdavis.edu/ knox/radpack.html
}

[1] A.G. Riess et al., Astrophys. J. 116, 1009 (1998).

[2] S. Perlmutter et al., Astrophys. J. 417, 565 (1999).

[3] A.G. Riess et al., Astrophys. J. 560, 49 (2001).

[4] C. Deffayet, Phys. Lett. B 502, 199 (2001).

[5] C. Deffayet, G. Dvali, and G. Gabadadze, Phys. Rev. D 65, 044023 (2002).

[6] G. Dvali, G. Gabadadze, and M. Porrati, Phys. Lett. B 485 , 208 (2000).

[7] G. Dvali and G. Gabadadze, Phys. Rev. D 63, 065007 (2001).

[8] G. Dvali, G. Gabadadze, M. Kolanovic, and F. Nitti, Phys. Rev. D 64, 084004 (2001).

[9] G. Dvali, G. Gabadadze, M. Kolanovic, and F. Nitti, Phys. Rev. D 65, 024031 (2002).

[10] G. Dvali, G. Gabadadze, X. Hou, and E. Sefusatti, hep-th/0111266.

[11] C. Deffayet, G.R. Dvali, G. Gabadadze, and A.I. Vainshtein, Phys. Rev. D 65, 044026 (2002).
[12] A. Lue, hep-th/0111168.

[13] A. Gruzinov, astro-ph/0112246.

[14] S.L. Adler, Rev. Mod. Phys. 54, 729 (1982); 55, 837(E) (1983).

[15] R. Dick, Class. Quantum Grav. 18, R1 (2001).

[16] A.A. Starobinsky, Phys. Lett. 91B, 9 (1980).

[17] R. Cordero and A. Vilenkin, Phys. Rev. D 65, 083519 (2002).

[18] R. Dick, Acta Phys. Pol. B 32, 3669 (2001).

[19] M. Goliath, R. Amanullah, P. Astier, A. Goobar, and R. Pain, Astron. Astrophys. 380, 6 (2001).

[20] P.P. Avelino and C.J. Martins, Astrophys. J. 565, 661 (2002).

[21] C. Deffayet, G. Dvali, and G. Gabadadze, astro-ph/0106449.

[22] U. Seljak and M. Zaldarriaga, Astrophys. J. 469, 437 (1996).

[23] C. Deffayet, A. Lue, and M. Zaldarriaga (in preparation).

[24] T. Shiromizu, K.i. Maeda, and M. Sasaki, Phys. Rev. D 62, 024012 (2000).

[25] P. Kraus, J. High Energy Phys. 12, 011 (1999). 
[26] P. Binetruy, C. Deffayet, U. Ellwanger, and D. Langlois, Phys. Lett. B 477, 285 (2000).

[27] E.E. Flanagan, S.H. Tye, and I. Wasserman, Phys. Rev. D 62, 044039 (2000)

[28] S. Mukohyama, T. Shiromizu, and K.i. Maeda, Phys. Rev. D 62, 024028 (2000); 63, 029901(E) (2000).

[29] F. Bonjour, C. Charmousis, and R. Gregory, Phys. Rev. D 62, 083504 (2000).

[30] D. Langlois, Phys. Rev. Lett. 86, 2212 (2001).

[31] D. Langlois, R. Maartens, M. Sasaki, and D. Wands, Phys. Rev. D 63, 084009 (2001).

[32] D. Langlois, Phys. Rev. D 62, 126012 (2000).

[33] H.A. Bridgman, K.A. Malik, and D. Wands, Phys. Rev. D 65, 043502 (2002)
[34] V.F. Mukhanov, H.A. Feldman, and R.H. Brandenberger, Phys. Rep. 215, 203 (1992).

[35] D. Wands, K.A. Malik, D.H. Lyth, and A.R. Liddle, Phys. Rev. D 62, 043527 (2000).

[36] A. Lue (private communication).

[37] M. Tegmark, M. Zaldarriaga, and A.J. Hamilton, Phys. Rev. D 63, 043007 (2001).

[38] X. Wang, M. Tegmark, and M. Zaldarriaga, Phys. Rev. D 65, 123001 (2002).

[39] N. Christensen, R. Meyer, L. Knox, and B. Luey, Class. Quantum Grav. 18, 2677 (2001).

[40] W.L. Freeman et al., astro-ph/0012376.

[41] M. Tegmark, astro-ph/0101354. 